\title{
Mechanical fracture characterization of alkali- activated slag mortars with standardized and natural sand
}

\author{
Hana Šimonová1,*, Barbara Kucharczyková ${ }^{1}$, Vlastimil Bílek, Jr. ${ }^{1,2}$ and Dalibor Kocáb ${ }^{1}$ \\ ${ }^{1}$ Brno University of Technology, Faculty of Civil Engineering, Veveří 331/95, 60200 Brno, Czech \\ Republic \\ ${ }^{2}$ Brno University of Technology, Faculty of Chemistry, Purkyňova 46/118, 612 00, Brno, Czech \\ Republic
}

\begin{abstract}
This paper is focused on the development of the physical, mechanical and fracture characteristics of alkali-activated slag (AAS) mortars during ageing. The two AAS mortars were selected for the purpose of the experiment. The difference was in a type of used sand: first mortar was made using the standardized siliceous sand with the maximum nominal grain size of $2 \mathrm{~mm}$ and the second mortar using the natural sand with a maximum grain size of $4 \mathrm{~mm}$. The results of the elastic, fracture and strength parameters determined within the time interval of 3 to 330 days of ageing are summarized in the paper. All experimental results were evaluated for the prismatic specimens with dimensions of $40 \times 40 \times 160 \mathrm{~mm}$ and were expressed by the average value and sample standard deviation calculated from three independent measurements.
\end{abstract}

\section{Introduction}

During recent decades, the research of various non-traditional binders as the modern alternatives to Portland cement has attracted great scientific attention. One wide group of these binders are alkali-activated materials (AAMs) whose setting and hardening processes are based on the dissolution of suitable amorphous aluminosilicate precursors in alkaline solution and condensation of the dissolved species into solid products, typically calcium/sodium-aluminate-silicate-hydrates, whose composition and properties strongly depend on the chemistry of both the precursor and the alkaline activator and as well as on curing conditions [1]. Alkali-activated slag (AAS) usually reaches high mechanical properties even at room temperature, but suffers from extensive shrinkage and cracking, particularly when subjected to drying, which has a negative impact on durability, mechanical parameters, etc.

Moreover, the cost of alkaline activators can also be an issue as well as their usually non-ecological production. The solution can be using some alternative activators. In this study, the waste sludge from water-glass production was applied. Because of significant amounts of dissolved alkalis and silicates, it belongs to dangerous waste and its disposal

* Corresponding author: simonova.h@ vutbr.cz 
is very expensive. On the other hand, it has a noticeable potential for alkaline activation. Moreover, it contains significant amounts of solid particles and the rest of the siliceous sand used for water-glass production, which would save natural fine aggregate. Therefore, this study is focused on mechanical fracture characterization of AAS mortars where this alternative activator was used for slag activation and simultaneously as a partially replacing material for standardized siliceous and natural sand.

\section{Experiments}

\subsection{Materials}

Ground granulated blast furnace slag with $90 \%$ content of amorphous phase and Blaine fineness of $400 \mathrm{~m}^{2} / \mathrm{kg}$ was used as a precursor for AAS mortars. The slag was activated by alternative activator - the waste sludge from water-glass production which contained $4.1 \% \mathrm{Na}_{2} \mathrm{O}, 7.3 \%$ soluble $\mathrm{SiO}_{2}, 16.5 \%$ water and $72.1 \%$ of non-reacted siliceous sand. The dose of activator was adjusted to $8 \% \mathrm{Na}_{2} \mathrm{O}$ with respect to the slag weight. Two sets of AAS mortars with sand to binder ratio of 3:1 were selected for the purpose of the experiment. The difference was in a type of used sand; first mortar was made using the standardized CEN siliceous sand with the maximum nominal grain size of $2 \mathrm{~mm}$ consisting of three fractions according to EN 196-1 [2] and the second mortar using the natural sand with a maximum grain size of $4 \mathrm{~mm}$. The type of sand was used to distinguish between the two mortar sets: $S$ (standard) and $N$ (natural). The water to slag $(w / s)$ ratio including water contained in the activator was 0.73 and 0.98 for $S$ and $N$ mixture, respectively. Such high values of $w / s$ ratio are caused by high water demand of the solid particles in the activator since they are relatively fine and porous. These solid particles introduced into the mixtures within the activator were also calculated to the dose of the above-mentioned sand to binder ratio, i.e. they partially replaced the used sands. For $S$ set, they replaced the whole finest fraction and a part of the middle one of the CEN sand, while for $N$ set its total amount was reduced accordingly. These differences in a replacement were the reason why the $w / s$ of the $N$ mortar was higher compared to that of $S$ mortar because in the latter case, the fine fraction was replaced by the fine solid part of activator which had a lower effect on $w / s$ than replacing the total amount of coarser sand. The particle size distribution of each sand is given in paper [3].

\subsection{Specimens production and curing conditions}

The Hobart mixer was used for the mixing of individual components and mixing took approximately 8 minutes. First, the alkali activator and water were mixed together to prepare a homogenous activating solution. Then, the slag and finally the sand was added. The mortars were cast into the polyethylene moulds (Hakorite) for preparation of prismatic specimens with nominal dimensions of $40 \times 40 \times 160 \mathrm{~mm}$ and sealed for 24 hours at a temperature of $21 \pm 2{ }^{\circ} \mathrm{C}$. Then the specimens were demoulded and stored in an airconditioned room where the temperature of $21 \pm 2{ }^{\circ} \mathrm{C}$ and relative humidity of $50 \pm 10 \%$ were maintained. In total, 15 specimens from each AAS mortar was prepared: 3 specimens were used for measurement of the length changes and 3 specimens were used for fracture tests at each investigated age of specimens. 


\section{Methods}

\subsection{Fracture tests}

The fracture parameters were determined at the age of 3,28, 107 and 330 days. The fracture characteristics were determined based on the load $F$ versus displacement $d$ (deflection in the middle of the span length) diagrams recorded during the three-point bending test of the prismatic specimens with an initial notch. The depth of the notch was, in this case, approx. 1/3 of the specimen's height. The span length was set to $120 \mathrm{~mm}$. The specimens were subjected to the quasi-static loading using a very stiff multi-purpose mechanical testing machine LabTest 6.250 with the load range of $0-250 \mathrm{kN}$ with a constant displacement increment of $0.02 \mathrm{~mm} / \mathrm{min}$ (see Fig. 1.).
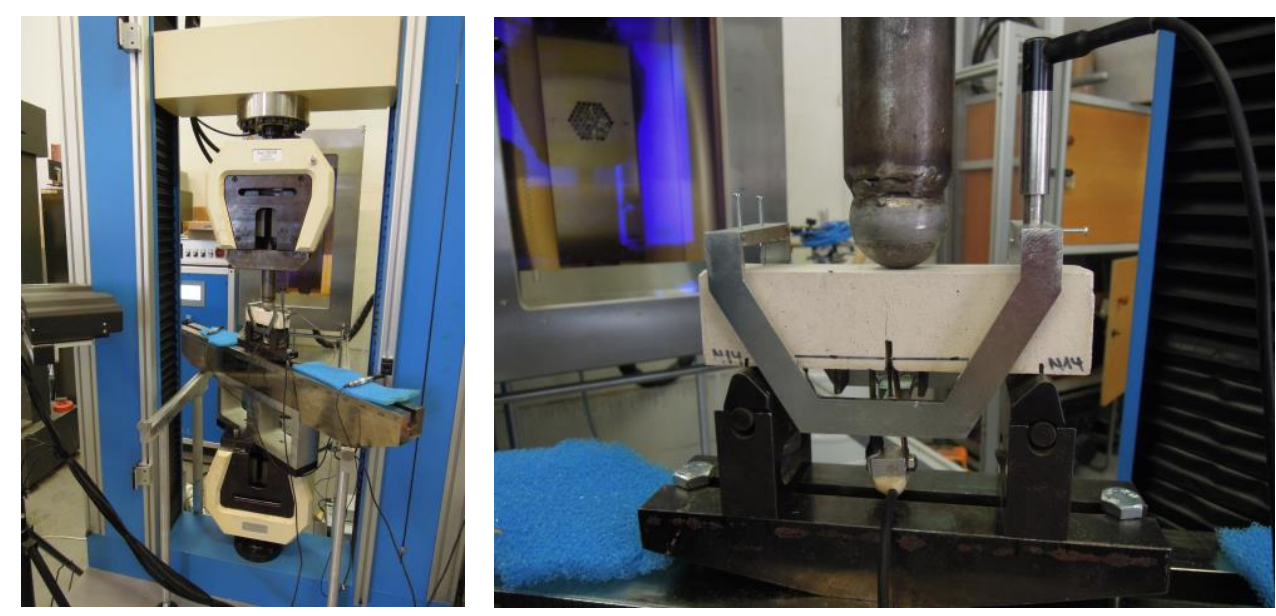

Fig. 1. Illustration of fracture test: overall view of testing machine (left), detail of measuring sensors positions.

At first, the measured diagrams were corrected in order to obtain the appropriate input values for subsequent diagrams evaluation using the selected fracture model. The corrected $F-d$ diagrams for both sets of specimens at investigated ages are introduced in Fig. 2 and 3. The initial parts of processed $F-d$ diagrams were used to estimate the modulus of elasticity value according to [4]. The fracture toughness value was determined using the effective crack model [4] which combines the linear elastic fracture mechanics and crack length approaches. The effective crack length, corresponding to the maximum load and matching mid-span displacement, had to be calculated first to determine the fracture toughness value. The work of fracture value was obtained from the whole $F-d$ diagrams according to the RILEM method [5] and corresponds to the area under the $F-d$ diagram. Subsequently, the specific fracture energy value was determined, that the work of fracture values were divided by the area of a ligament (cross-section of specimens through which the crack grows).

The informative compressive strength value was determined on the fragments remaining after the fracture experiments of AAS composites had been performed. 

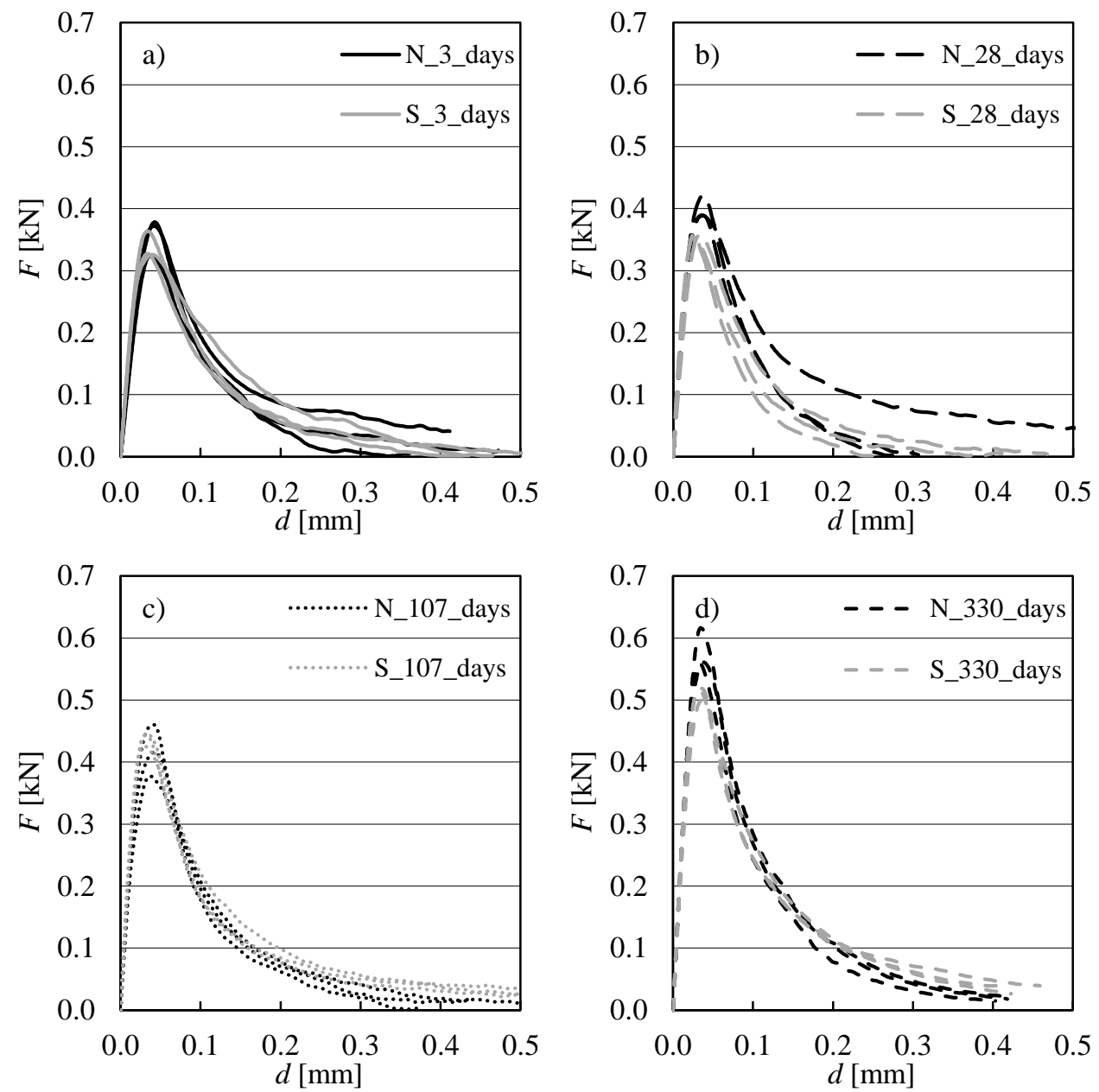

Fig. 2. The processed $F-d$ diagrams for both sets of AAS mortars at investigated ages of specimens: a) 3 days, b) 28 days, c) 107 days, d) 330 days.

\subsection{Resonance method}

During the specimens ageing, the dynamic modulus of elasticity was monitored and determined using the resonance method according to the standard ČSN 731372 [6]. The natural frequency of longitudinal vibration was determined for each specimen. The vibration was generated by a mechanical impulse using the impact hammer and the natural frequency was measured by an oscilloscope Handyscope HS4 with a piezoelectric sensor. The dynamic modulus of elasticity $E_{\text {dyn }}$ was then calculated from this natural frequency according to the standard [6].

\subsection{Shrinkage measurement}

The shrinkage process of the AAS mortars was determined based on the measurement of the length changes of the prismatic specimens equipped with the special markers embedded into the ends of the test specimens (in the longitudinal axis of the specimens). The measurement was started right after the demoulding of the test specimens at the age 
of 24 hours. The changes in length were measured in regular intervals using the measurement gauge equipped with the digital probe with a resolution of $0.001 \mathrm{~mm}$. Details about the development of shrinkage values in time can be found in [3].

\section{Results}

The average values (determined based on 3 independent measurements) and sample standard deviations (given by the error bars) of the monitored parameters of AAS mortars determined using the above-described non-linear fracture models from $F-d$ diagrams are summarized in the Figs. 3-5. The mechanical fracture parameters were monitored during the specimens ageing at the age of 3,28, 107 and 330 days.

The modulus of elasticity values gradually increased with the specimen's age for both mortar sets (see Fig. 3). The modulus of elasticity achieved the highest values of about $10 \mathrm{GPa}$ at the age of 330 days. When the sample standard deviation determined for the particular ages is taken into account for the evaluation of results, it can be stated, that both mortars achieved almost the same values of modulus of elasticity within the time interval from 28 to 330 days. The highest difference between both mortars was observed at the age of 3 days. This can be attributed to the higher $w / s$ ratio of mortar set $N$ which slowed the initial growth of the modulus of elasticity.

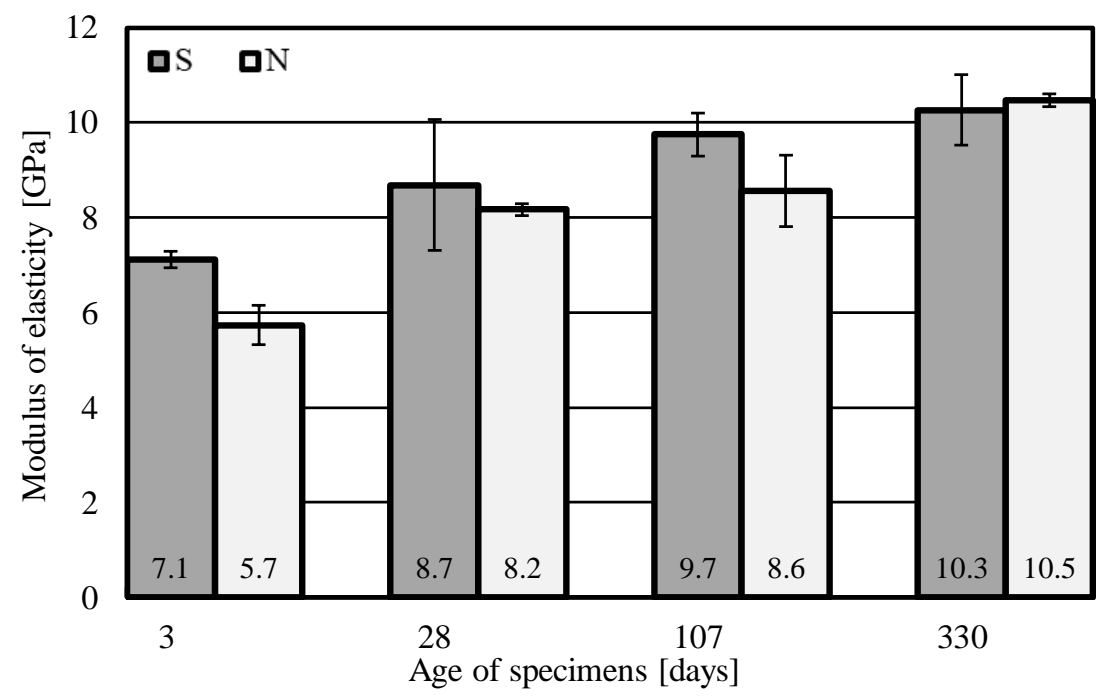

Fig. 3. The modulus of elasticity values at investigated ages of specimens.

The effective fracture toughness values also gradually increased with the specimen's age for both mortar sets (see Fig. 4). The fracture toughness increased by 45 and $28 \%$ for mortar set $S$ and $N$, respectively, when the values are compared at the age of 28 and 330 days. 


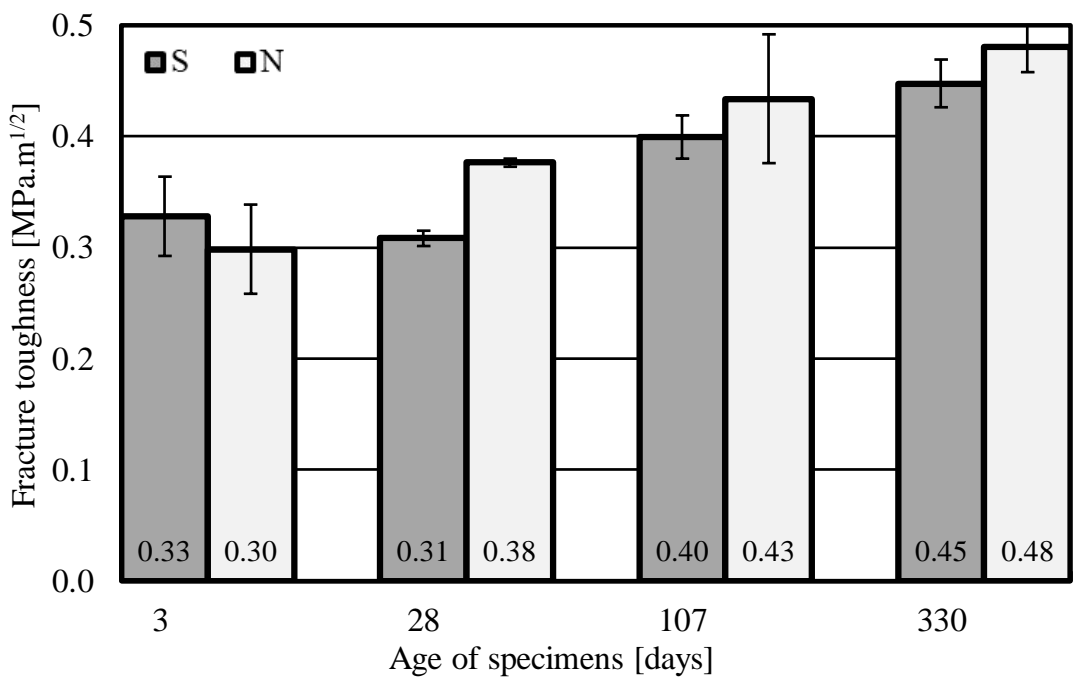

Fig. 4. The effective fracture toughness values at investigated ages of specimens.

The specific fracture energy value gradually increased with the specimen's age for mortar set $N$. The highest value $66 \mathrm{~J} / \mathrm{m}^{2}$ was achieved at the age of 330 days and it is about $37 \%$ higher in comparison with value at the age of 28 days. In the case of mortar set $S$, the fracture energy value decreased between the age of 3 and 28 days. After that, the gradual increase with specimen's age was also monitored. The increase was about $63 \%$ when the values are compared at the age of 28 and 330 days (see Fig.5).

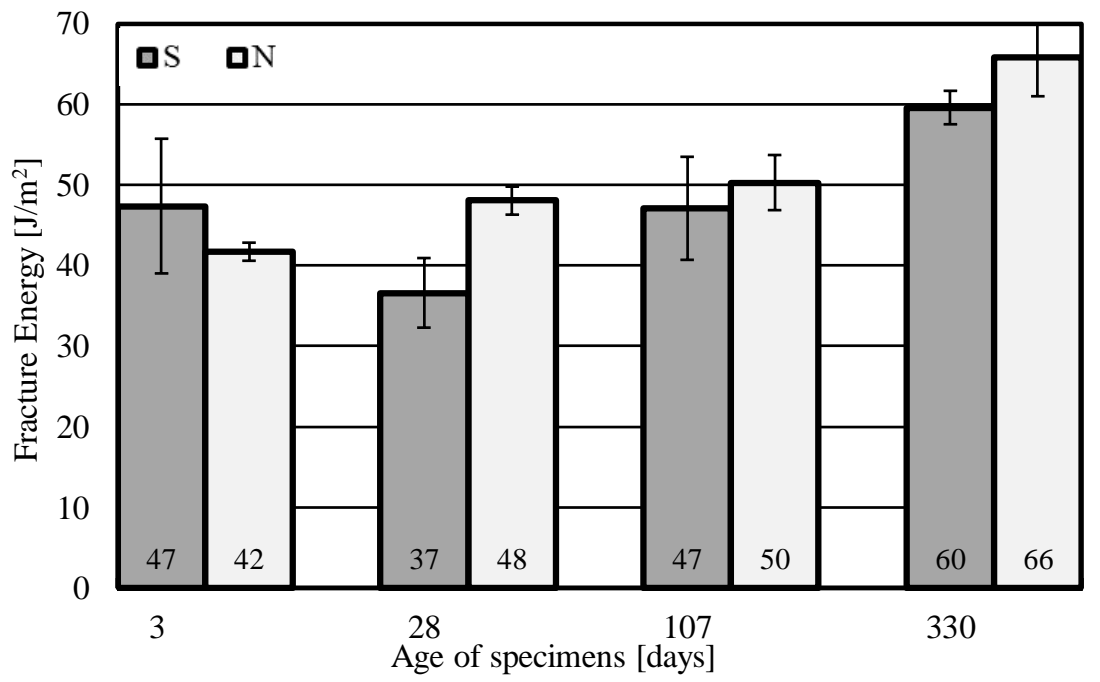

Fig. 5. The specific fracture energy values at investigated ages of specimens.

The informative compressive strength value gradually increased with the specimen's age for both mortar sets. The increase is not so significant during the time interval between 28 and 330 days as in the case of other monitored mechanical fracture parameters (see Fig. 6). 


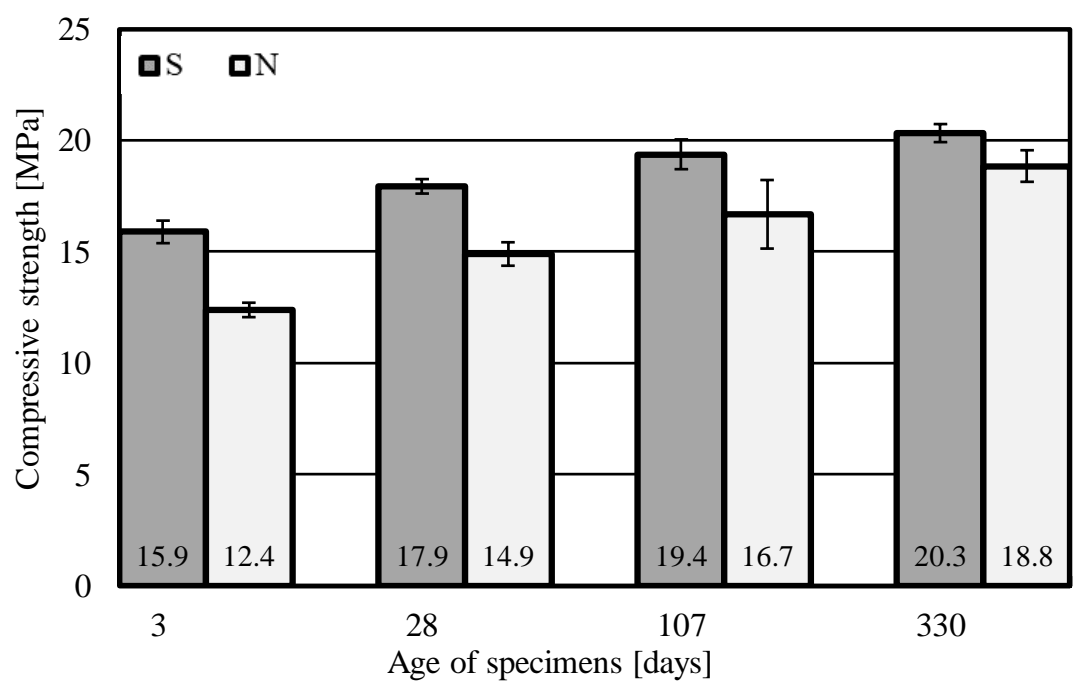

Fig. 6. The informative compressive strength values at investigated ages of specimens.

Additionally, during the specimens ageing at the age of 2, 27, 93 and 178 days, the dynamic modulus of elasticity determined using the resonance method was also monitored (see Fig. 7). The significant increase of dynamic modulus of elasticity was achieved within the time interval 2 and 27 days for both mortar sets. In the case of mortar set $N$, the value was about 3 times higher when the values are compared at the age of 2 and 27 days. The trend of increase of the value after the age of 28 days is similar as in the case of modulus of elasticity determined from $F-d$ diagrams (see Fig. 3 ).

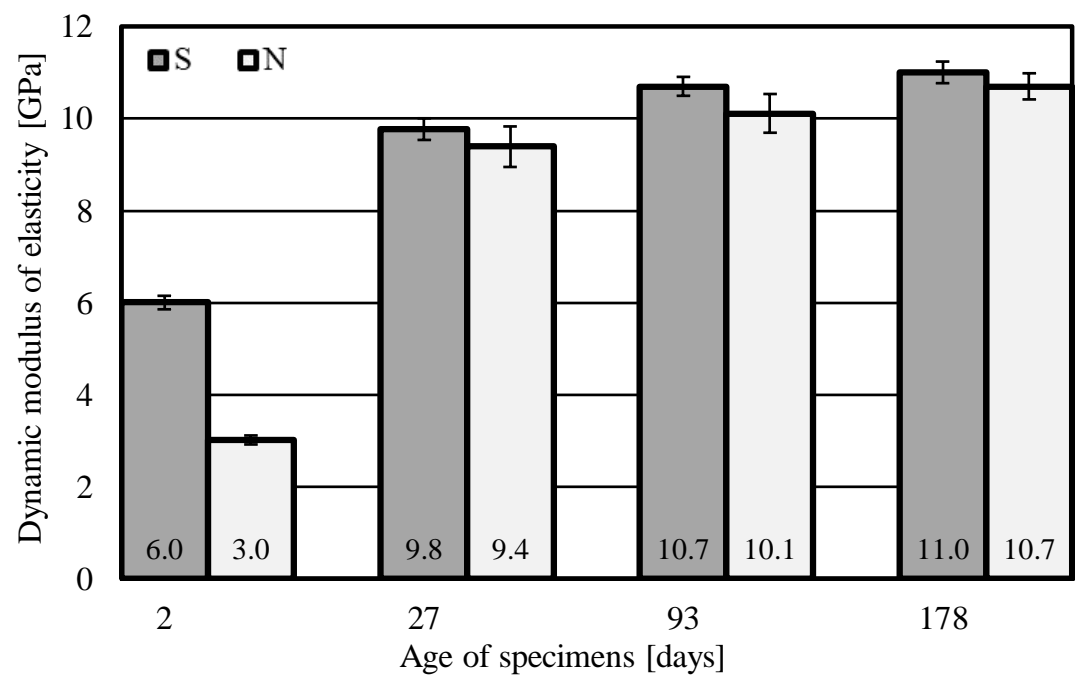

Fig. 7. The dynamic modulus of elasticity values at investigated ages of specimens. 


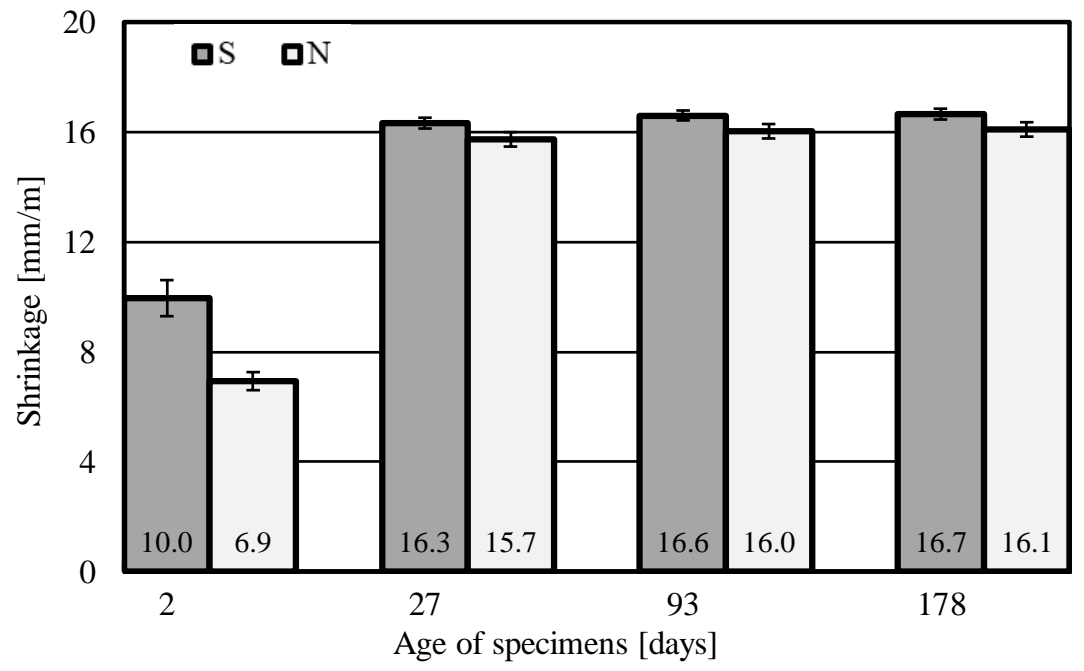

Fig. 8. The shrinkage values at investigated ages of specimens.

For illustration, the shrinkage values measured at the same ages of specimen's as dynamic modulus of elasticity are shown in Fig. 8. Details about the development of shrinkage values during the whole time interval of measurement can be found in [3]. The trend of development of shrinkage during the specimen's ageing is similar to the development of dynamic modulus of elasticity. In spite of higher $w / \mathrm{s}$ of mortar set $N$, the initial shrinkage recorded was lower by about $30 \%$ compared to the mortar set $S$. This effect can be attributed to the different maximum grain size of the aggregate which was 4 and $2 \mathrm{~mm}$ for mortar set $N$ and $S$, respectively. It can be supposed, that larger grains of aggregate diminished the steepness of the initial growth of the shrinkage. There was not observed so significant effect of aggregate grain size on the shrinkage values in later ages.

\section{Conclusions}

The results of the pilot study of two sets of mortars made from slag activated by the waste sludge from water-glass production were presented in this paper. The high water demand of the waste sludge due to the relatively high content of fine and porous particles is one of the main disadvantages of these materials which results in high values of $w / s$ ratio because of necessity to achieve appropriate workability of the mortars during the manufacturing process.

The high $w / s$ ratio affect negatively the process of desiccation and shrinkage. The shrinkage of mortar set $S$ at the age of 28 and 90 days is more than 2 times higher in comparison with AAS mortar activated by water-glass with same sand to binder ratio but lower $w / s$ ratio [7]. The process of desiccation is also accompanied by the formation of cracks, which affects the mechanical fracture parameters. Also monitored mechanical fracture parameters of mortar set $S$ at the age of 90 days are lower in comparison with previously investigated AAS mortar activated by water-glass [7].

The results of this study serve as the basis of the ongoing research which will be focused especially on the ways for decreasing the $w / s$ and shrinkage value at the preservation of required workability during the manufacturing process, which should lead also to the increase of mechanical fracture parameters. 
This outcome has been achieved with the financial support of the Czech Science Foundation under project No 18-12289Y.

\section{References}

1. J.L. Provis, J.S.J Van Denvenetr, eds, Alkali activated materials: state-of-the-art report, RILEM TC 224-AAM (Springer, Dordrecht, 2014)

2. ČSN EN 196-1, Methods of testing cement - Part 1: Determination of strength (ÚNMZ, Prague, 2016)

3. B. Kucharczyková, V. Bílek, Jr., H. Šimonová, IOP Conf. Ser.: Mater. Sci. Eng. 660, 012011 (2019)

4. B.L. Karihaloo, Fracture Mechanics and Structural Concrete (Longman Scientific \& Technical, New York, 1995)

5. RILEM TC-50 FMC Recommendation, Mater. Struct. 18, 285-290 (1985)

6. ČSN 73 1372, Non-destructive testing of concrete - Testing of concrete by resonance method (ÚNMZ, Prague, 2012)

7. V. Bílek, Jr., B. Kucharczyková, H. Šimonová, D. Kocáb, Z. Keršner, IOP Conf. Ser.: Mater. Sci. Eng. 379, 012018 (2018) 